\title{
Efficient near-infrared supercontinuum generation in tellurite holey fiber pumped 320nm within the normal dispersion regime
}

\author{
Jindan Shi, Xian Feng, Kangkang Chen, Peh Siong Teh, Peter Horak, Dejiao Lin, Shaif-ul Alam, \\ Wei H. Loh, David J. Richardson, Morten Ibsen \\ Optoelectronics Research Centre, University of Southampton, Southampton, SO17 IBJ, UK \\ ixs@orc.soton.ac.uk
}

\begin{abstract}
We demonstrate the generation of a $1.1-1.7 \mu \mathrm{m}$ supercontinuum in a $1.3 \mathrm{~m}$ long airsuspended-core tellurite holey-fiber with a nominal zero-dispersion wavelength of $\sim 1.38 \mu \mathrm{m}$. The fiber is pumped at $1.06 \mu \mathrm{m}$ with a $20 \mathrm{ps}$ pulsed fiber laser. The high Raman gain-coefficient of tellurite glass is attributed a key role in the supercontinuum generation.
\end{abstract}

\section{Introduction}

Broadband 0.35-5 $\mu \mathrm{m}$ infrared supercontinuum (SC) sources [1] with power spectral densities in the $\mathrm{mW} / \mathrm{nm}$ range are highly desirable for applications in for example, eye-safe airborne light detection and ranging (LIDAR), electronic countermeasures (ECM), optical coherence tomography (OCT), metrology, and IR spectroscopy. Commonly, SC is generated by pumping near the zero dispersion wavelength, or slightly into the anomalous dispersion regime of the fiber. The spectral broadening is due to a combination of nonlinear effects within the fiber including modulation instability and soliton-related dynamics [2]. One slight issue related to the generation of SC is that the pump wavelength should be relatively well matched to the dispersion zero of the fiber, which at times can impose a limitation on SC generation in more exotic fibers due to difficulties with matching the wavelength of available pump lasers with the zero dispersion wavelength of these fibers. In this work, we demonstrate the generation of near-infrared SC in a tellurite fiber by pumping in the normal dispersion regime more than 300nm away from the dispersion zero. We believe that the ultra-wide and intensive Raman gain profile of the fiber plays a key role in facilitating the generation of the supercontinuum.

\section{Experimental results and discussions}

A tellurite glass air-suspended-core (ASC) holey fiber (HF) was used in this experiment. The glass composition is $75 \mathrm{TeO}_{2}-20 \mathrm{ZnO}-5 \mathrm{Na}_{2} \mathrm{O}$ (mol. \%) $(\mathrm{TZN})$, and the fiber preform was made using an in-house extrusion technique [3]. Fig. 1(a) shows the cross section of the fiber which has an effective mode area of $2.6 \mu \mathrm{m}^{2} @ 1.06 \mu \mathrm{m}$. The fiber is single-moded at $1.06 \mu \mathrm{m}$, and the calculated dispersion profile, shown in Fig. 1(b), reveals the zero dispersion wavelength (ZDW) to be $\sim 1.38 \mu \mathrm{m}$ and the nominal dispersion to be $-320 \mathrm{ps} / \mathrm{nm} / \mathrm{km} @ 1.06 \mu \mathrm{m}$. The propagation loss of the fiber at $1.06 \mu \mathrm{m}$ is measured to be $2.3 \pm 0.2 \mathrm{~dB} / \mathrm{m}$ determined by the cut back method.

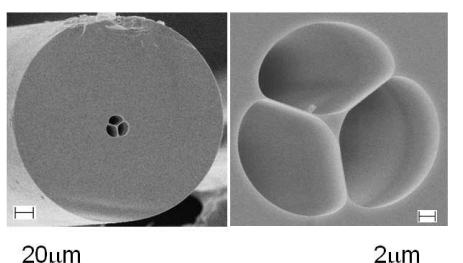

(a)

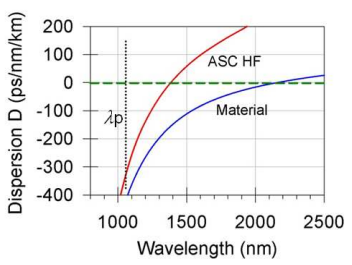

(b)

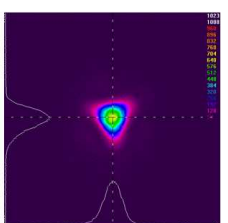

$@ 1.06 \mu \mathrm{m}$

(c)

Fig. 1: (a) SEM (Scanning Electron Microscope) photographs of fabricated tellurite ASC HF with 2.6 $\mu$ m core diameter; (b) Calculated dispersion curves of fabricated tellurite ASC HF and TZN bulk glass; The dotted vertical line indicates the pump wavelength; (c) Observed nearfield mode profile from the fiber at $1.06 \mu \mathrm{m}$.

The experimental setup consists of a $1.06 \mu \mathrm{m}$ Yb-doped fiber laser source with a pulse duration of 20ps and a repetition rate of $14.4 \mathrm{MHz}$. The source is launched into the small core of the tellurite HF through a sequence of free-space optics to ensure a good overlap with the core [4], and a CCD camera is used to monitor the coupled pump intensity in the core (see Fig. 1(c)). The coupling efficiency, defined as the ratio of the power launched into the HF to the input pump power before the lens, was measured to be $39 \%$. The generated output spectra from the 
fiber were recorded with an OSA with the resolution bandwidth of $0.5 \mathrm{~nm}$. For a more precise assessment of the generated average powers, we used a power meter to measure the average power at the output end of the HF.

The generated Stimulated Raman Scattering (SRS) and SC spectra from the HF are shown in Fig. 2(a). When the launched average pump power is below $78.6 \mathrm{~mW}$, corresponding to a peak power of $273.1 \mathrm{~W}$, only discrete and cascaded Raman Stokes spectra and no supercontinuum is observed. This is believed to be due to the fact that only the Raman Stokes components are being generated in this power range since the pump wavelength is well within the highly normal dispersion regime of the HF. The high dispersion at the pump wavelength also implies that fourwave mixing (FWM) is largely suppressed by phase mismatch and walk-off effects [2]. Specifically, the first-order Stokes component with two peaks was clearly observed when the launched average pump power was fairly low at just $16.5 \mathrm{~mW}$. These two Raman bands at $1.11 \mu \mathrm{m}$ and $1.15 \mu \mathrm{m}$ arise from the Te-O-Te chain unit's symmetric stretching mode with a Raman shift of $440 \mathrm{~cm}^{-1}$ and a $\mathrm{TeO}_{4}$ bipyramidal unit with a Raman shift of $740 \mathrm{~cm}^{-1}$, respectively [5]. When the launched average pump power was increased to $35.6 \mathrm{~mW}$, the second-order Stokes component peaking at $1.25 \mu \mathrm{m}$ began to appear. By increasing the launched pump power further the spectrum broadened into a more continuous profile due to cascaded SRS effects. For a launched average pump power of $104.4 \mathrm{~mW}$ (corresponding to the peak power of $362.5 \mathrm{~W}$ ), a broad SC spectrum from around $1.1 \mu \mathrm{m}$ to above $1.7 \mu \mathrm{m}$ was clearly observed (careful investigations of the wavelength band above $1700 \mathrm{~nm}$ was limited by the range of the OSA). We believe that as the higher order Stokes components are being generated for increasing pump powers they eventually act as the pump for the SC once the ZDW point of the HF is reached $[2,6]$.

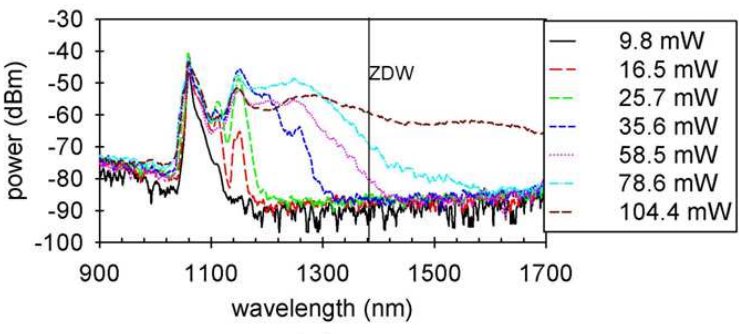

(a)

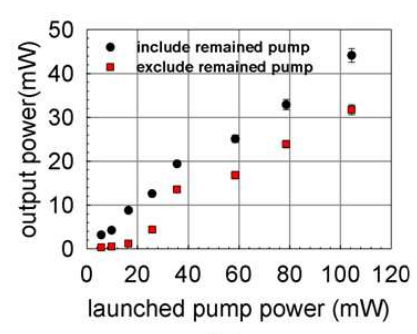

(b)

Fig. 2: (a) SRS-assisted SC spectra from 1.3m-long tellurite ASC HF under different launched average pump powers; The solid vertical line indicates the ZDW of the HF; (b) Average output powers vs launched average pump powers

Fig. 2(b) shows the relation between the average output power including and excluding the residual pump at $1.06 \mu \mathrm{m}$ against the launched average pump power. The average output power excluding the remained pump is calculated by numerical integration of the continuum output spectrum shown in Fig. 2(a). The highest output power is limited by the damage at the input facet of the HF. We observed that the core of the fiber at the input end melted when the launched average pump powers went above $\sim 140 \mathrm{~mW}$. The reason behind this melting is currently under investigation.

\section{Conclusions}

An efficient, broad and flat SC has been generated between 1.1-1.7 $\mu \mathrm{m}$ from a $1.3 \mathrm{~m}$ long tellurite ASC HF using a 20ps pulsed laser with a wavelength $320 \mathrm{~nm}$ below the ZDW. We attribute the generation of the SC to the combination of the broad and high Raman intensity of the tellurite glass, and the small core and high nonlinearity of the ASC HF. The conversion efficiency from the pump to the SC is measured to be more than $30 \%$. We believe that the high Raman gain coefficient and large Raman shift of tellurite glass promises well for short-length tellurite ASC HF based Raman active medium for infrared applications.

\section{References}

[1] R. R. Alfano (Editor), “The Supercontinuum Laser Source: Fundamentals with Updated References”, Springer, 2nd edition, (2005).

[2] J. M. Dudley, et al, "Supercontinuum generation in photonic crystal fiber", Reviews of Modern Physics, 78(4), pp.1135-1184, (2006).

[3] X. Feng, et al, "Extruded singlemode, high-nonlinearity,tellurite glass holey fibre," Electronics Letter, 41(15), pp. 835-837, (2005).

[4] K.K.Chen, et al, "Picosecond fiber MOPA pumped supercontinuum source with 39 W output power", Optics Express, 18(6), pp. 5426-5432, (2010).

[5] Robert Stegeman, et al, "Tellurite glasses with peak absolute Raman gain coefficients up to 30 times that of fused silica", Optics Letters, 28(13), pp. 1126-1128, (2003).

[6] A. K. Abeeluck and C. Headley, "Continuous-wave pumping in the anomalous- and normal-dispersion regimes of nonlinear fibers for supercontinuum generation", Optics Letters, 30(1), pp. 61-63, (2005). 